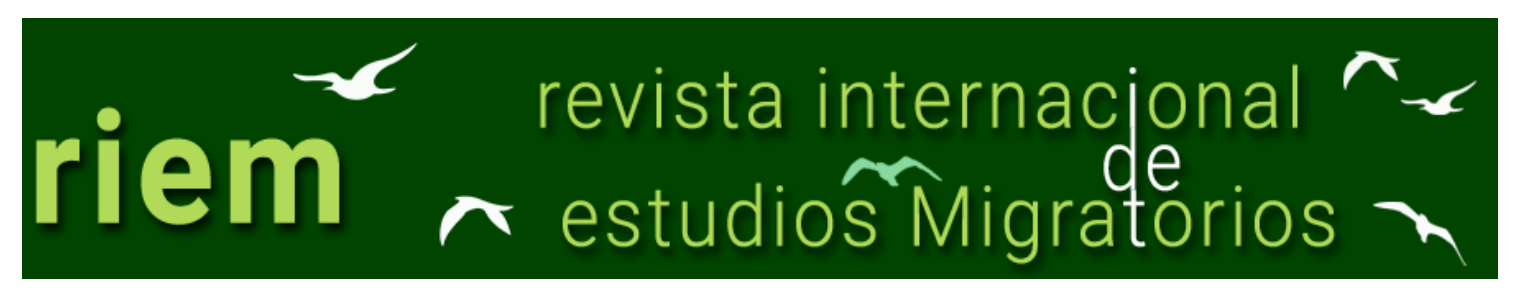

ISSN: 2173-1950

\title{
Europa/crisis. Nuevas palabras clave en la 'crisis' en y de 'Europa'. De Genova, N. y Tazzioli, M. (Eds.) (2021). Madrid: Los Libros de la Catarata.
}

José Antonio Abreu Colombrí1

La publicación colectiva, esencialmente, se centra en las consecuencias de la llegada masiva de refugiados para el conjunto de la Unión Europea, también en el análisis de los escenarios geo-estratégicos y los condicionantes político-diplomáticos. La transformación de las estrategias de gestión migratoria de los Estados miembros, a raíz de la crisis de 2015 (ruta de las islas Egeas), ha modificado totalmente la agenda común y el proceso legislativo y administrativo. Los fenómenos migratorios masivos tuvieron un gran impacto sobre la opinión pública a mediados de la década pasada, pero la constante presencia mediática de las crisis migratorias ha horadado la sensibilidad colectiva y ha soliviantado a la clase política europea (de muy diferentes formas). Son muy clarificadoras algunas líneas del prólogo (Soledad Álvarez Velasco, Maribel Casas-Cortés, Sebastián Cobarrubias y Nicholas De Genova):

“[...] Si en el 2015 los esfuerzos de cruzar el mar Egeo y el 'corredor de los Balcanes' fueron el foco de atención político-mediática, en seguida el Mediterráneo central volvió a ser el protagonista de la mirada securitariahumanitaria dado el número en aumento de naufragios. En el 2017 hay un repunte en los intentos de saltar las rejas de Ceuta y Melilla, con su consiguiente represión, para que en los últimos meses del 2020 centrar la

\footnotetext{
${ }^{1}$ Doctor por la Universidad de Alcalá, Alcalá de Henares, España. abreucolombri@gmail.com 
atención en las islas Canarias de nuevo [...] la palabra crisis es ya una recurrencia desde hace décadas para hablar de los sucesos en varios puntos fronterizos a lo largo de la UE [...]” (página 10).

La gran repercusión del libro, coordinado por Nicholas De Genova² y Martina Tazzioli3, radica en la acertada crítica del contexto socio-político actual de toda la región europea. Ambos autores dan forma a una interesante secuencia descriptiva de los acontecimientos, desde 2015 hasta la actualidad, en materia legislativa, mediática e ideológica. Puntualmente, se traza un marco comparativo, circunspecto y riguroso, entre el caso de estudio europeo y las dinámicas migratorias de la región norteamericana.

Europa / crisis. Nuevas palabras clave de 'la crisis' en y de 'Europa'4 es una investigación colectiva, que se estructura a través de siete capítulos. En la parte final del libro hay un apartado general de fuentes documentales (con libros, artículos, reseñas, informes e interpretaciones legales) y la lista de autores participantes (con información académica, formativa y bibliográfica).

El primer capítulo, con título homónimo, está realizado por los dos coordinadores de la publicación. En estas páginas se hace un repaso a los diferentes conceptos surgidos en el contexto académico sobre la sucesión de crisis humanitarias en las fronteras de la Unión Europea, mediante un análisis de los fenómenos migratorios y de la gestión de las autoridades migratorias a nivel político y administrativo (Bruselas y FRONTEX5).

“Crisis” es el título del segundo capítulo. Los autores tratan de establecer los límites retóricos del término "crisis" en los ámbitos mediático y académico. Se pone el foco sobre los esfuerzos de las autoridades diplomáticas europeas por internacionalizar el problema migratorio, para que países colindantes se involucren en la defensa fronteriza de la Unión Europea. También se presta una especial atención a la disrupción general surgida de la

\footnotetext{
2 Es director del Departamento de Estudios Culturales Comparados de la Universidad de Houston. Ha sido profesor contratado y visitante de más de una decena de universidades en Holanda, Suiza, los Estados Unidos y el Reino Unido. Tiene multitud de libros y proyectos de investigación relacionados con la inclusión social, la legislación migratoria, el proceso de asilo y el impacto cultural de la inmigración.

3 Es profesora del Departamento de Política y Tecnología de la Universidad de Londres (campus de Goldsmiths). Es una destacada estudiosa de los procesos migratorios del sur de Europa y sus efectos sobre la opinión pública, también está especializada en el proceso mediático de subjetivación de la información y los efectos psicológicos de las nuevas tecnologías de la comunicación. Forma parte del consejo de redacción de la revista Radical Philosophy.

4 Los Libros de la Catarata es uno de los grupos más comprometidos con las grandes problemáticas sociales del momento presente. A nivel de impacto científico, la editorial madrileña lleva una estela ascendente desde hace más de una década. Sus diferentes colecciones están cada vez más consolidadas comercialmente y sus espacios de distribución no paran de expandirse.

5 Agencia Europea de la Guardia de Fronteras y Costas.
} 
“Primavera Árabe” y las asimetrías geográficas del Viejo Continente. En el capítulo participan: Charles Heller, Maurice Stierl, Huub van Baar y los dos coordinadores.

El tercer capítulo, “Crisis de migrantes' / 'crisis de refugiados”, se centra en el proceso de solicitud de asilo y refugio y los diferentes estatus de la población migrante en el contexto legislativo internacional. Elaborado por Elena Fontanari, Fiorenza Picozza, Laia Soto Bermant, Aila Spathopoulou, Maurice Stierl, Zakeera Suffee, Huub van Baar, Can Yildiz y los dos coordinadores. La migración se ha convertido en uno de los temas más manidos en las redacciones periodísticas durante la última década, porque representa un tema muy sensible para la sociedad (por muy diferentes y contrapuestas razones) y una fuente de estereotipia política (en todas las regiones europeas), especialmente en los Estados miembros receptores de los flujos migratorios. Se lleva a cabo un repaso de los principales elementos del debate político entre socios, de las motivaciones de la falta de acuerdo en materia migratoria y de las diferencias socioeconómicas entre regiones para afrontar el reto de la inmigración masiva.

"Cifras (o el espectáculo de la estadística de la producción de la crisis)" es el ilustrativo título del cuarto capítulo (Maurice Stierl, Charles Heller y Nicholas De Genova). En este capítulo, bajo el prisma de la academia, se desarrollan algunas reflexiones sobre el uso de los datos estadísticos de forma interesada, por parte de los Estados miembros, para tratar de dar prioridad a cuestiones concretas en la agenda común. La "matematización" del problema migratorio está interrelacionada con otras cuestiones de primer nivel, como el problema de la deuda, el paro estructural de la región sur, la tendencia del sistema impositivo y los criterios de designación presupuestaria.

El capítulo quinto se titula “'Crisis humanitaria”" (Elena Fontanari, Irene Peano, Maurice Stierl y los dos coordinadores). En estas páginas se pretende arrojar luz sobre las calamidades sufridas por los grupos de población migrante, antes, durante y después del tránsito hacia el destino europeo. También se destaca la existencia de un colectivo muy amplio de activistas, académicos, periodistas y abogados que lucha por los derechos de las personas que solicitan asilo y refugio, tratando de contrarrestar las ideas de negatividad que envuelven a las dinámicas migratorias: ilegalidad, insalubridad, inseguridad, delincuencia, tráfico de personas, terrorismo, inadaptación, etcétera.

El espacio de libre movilidad se ha convertido en causa de orgullo y conflicto entre varias generaciones de líderes europeos. Es una cuestión de orgullo por ser uno de los pasos más firmes en el proyecto de unificación política, pero, al mismo tiempo, es una cuestión muy 
conflictiva, por la gestión de los campos de retención de inmigrantes, la tramitación de las deportaciones y la designación de las cuotas de refugiados. En estas páginas se menciona el malestar creciente de la ciudadanía respecto a la llegada de inmigrantes, por cuestiones relacionadas con la falta de oportunidades laborales o los discursos xenófobos y racistas, pero no se presta la suficiente atención a los discursos de odio y al auge de las ideologías ultraderechistas. La libertad de circulación se veía hace décadas como un logro, y ahora se plantea como una causa de debilidad. El fomento de la movilidad de los estudiantes y los trabajadores tienen un reverso oscuro, alimentado por la "migrantización" de las prácticas y los planes para la movilidad ciudadana. El sexto capítulo: "Movilidad” (Lisa Riedner, Soledad Álvarez-Velasco, Huub van Baar y los dos coordinadores).

Con motivo del tema de los valores europeos (derechos humanos, principios democráticos y programas de inclusión) y a la proyección de un poder blando de las instituciones de la Unión Europea, hay un párrafo que merece ser transcrito por su análisis sincrético:

“[...] el europeísmo de hoy se basa en la sorprendentemente superficial reconstrucción de la historia 'europea' como un asunto insular y herméticamente sellado. Por supuesto, en medio de la actual proliferación de discursos ideológicos arrogantes sobre la cultura, la civilización, la identidad y los valores europeos, los ultrajes abiertamente racistas de los populismos neofascistas y de extrema derecha simplemente saca a la luz y hacen explícito lo inextricable que es cualquier europeísmo de la difusión de la europeidad como una formación racial blanca, incluso cuando esta viene disimulada enfática y aparentemente en favor de las construcciones identitarias en torno a las diferencias ‘culturales'o ‘de las civilizaciones' [...]” (página 98).

"(La crisis de) los 'valores europeos" (capítulo séptimo) establece un juego gramatical con el título, para dar a entender la gravedad de la falta de valores y de consenso en la política exterior común de los socios europeos. Los aspectos más filosóficos y ensayísticos del libro están contemplados aquí. El euroescepticismo aumenta tanto en los sectores conservadores como en los progresistas; la cuestión migratoria polariza a la sociedad coyunturalmente. Las críticas contenidas en estas páginas son obra de Can Yildiz, Nicholas De Genova, Yolande Jansen, Laia Soto Bermant, Aila Spathopoulou, Maurice Stierl y Zakeera Suffee.

La principal aportación de Europa/crisis. Nuevas palabras clave de 'la crisis' en y de 'Europa', en relación con la cuestión del desastre humanitario, comporta la denuncia de las 
graves consecuencias de la inacción política en materia de prevención de desastres, que conllevan la desaparición y la muerte de seres humanos. La ausencia de un apartado de conclusiones generales no dificulta la asimilación de las ideas principales del texto, ya que la mayoría de los capítulos, en su parte final, tienen rasgos ensayísticos, que enfatizan mucho el punto de vista y los fundamentos teóricos de los autores participantes.

De Genova, N. y Tazzioli, M. (Eds.) (2021). Europa / crisis. Nuevas palabras clave en la 'crisis' en y de 'Europa'. Madrid: Los Libros de la Catarata, 126 páginas. ISBN: 978-84-1352$155-8$ 